\title{
Constellation Design for Widely Linear Transceivers
}

\author{
Maddalena Lipardi, ${ }^{1}$ Davide Mattera, ${ }^{1}$ and Fabio Sterle ${ }^{2}$ \\ ${ }^{1}$ Dipartimento di Ingegneria Elettronica e delle Telecomunicazioni, Università degli Studi di Napoli Federico II, via Claudio 21, \\ 80125 Napoli, Italy \\ ${ }^{2}$ Dipartimento di Sistema Radar, Selex Sistemi Integrati, Via Giulio Cesare 105, 80070 Bacoli (NA), Italy
}

Correspondence should be addressed to Davide Mattera, mattera@unina.it

Received 31 October 2009; Revised 3 May 2010; Accepted 6 July 2010

Academic Editor: Ananthram Swami

Copyright ( $) 2010$ Maddalena Lipardi et al. This is an open access article distributed under the Creative Commons Attribution License, which permits unrestricted use, distribution, and reproduction in any medium, provided the original work is properly cited.

Constellation design has been previously addressed by assuming that there is a linear equalizer at the receiver side. However, the widely linear equalizer is well known to outperform the linear one with no significant complexity increase; we derive optimum and suboptimum techniques for constellation design in presence of such an equalizer. The proposed techniques adapt the circularity properties of the transmitted signals to the specific channel to be equalized; their performance analysis shows that also the simplest suboptimum procedure provides significant improvements over a fixed-constellation scheme.

\section{Introduction}

Constellation design has been previously addressed by assuming that there is a linear equalizer at the receiver side. In early works (see, e.g., $[1,2]$ ), the optimization of a two-dimensional constellation in order to minimize the symbol error rate (SER) was first addressed with reference to the transmission over a nondispersive channel affected by additive noise.

The advantage provided by the constellations with two degrees of freedom (such as quadrature amplitude modulation (QAM)) over the ones with one degree of freedom (such as phase-shift-keying (PSK), and pulse amplitude modulation (PAM)) was shown [1], and a proper mapping (based on a gradient-descent procedure) of the $\log _{2} K$ information bits into $K$ points of a two-dimensional constellation was proposed [2]. However, the adoption of an additivenoise nondispersive channel model allows one to consider the constellation mapping independently of the equivalent channel. On the other hand, an amount of literature (e.g., [3-7]) refers to the optimization of the transmitter and/or the receiver without including the choice of the constellation in the optimization procedure. In fact, many existing transceiver processing techniques are optimized (according to a chosen criterion) by only exploiting knowledge of the statistics of the information symbol sequence.
This paper addresses the constellation design under the assumption that the transmitter is fixed (i.e., by considering an equivalent channel representing the transmitter and the channel) and a widely linear (WL) minimum mean square error (MMSE) equalizer is employed at the receiver side [8$14]$.

The WL filtering generalizes the conventional linear filtering and allows one to achieve a power reduction of the additive noise and interferences at the equalizer output, and therefore a performance gain, by exploiting the statistical redundancy possibly exhibited by a rotationally variant transmitted (and/or received) signal. For such a reason, the adoption of the WL equalization has frequently been confined to the transmission of one-dimensional constellations (see, e.g., [3, 15-17] and references therein) since the advantage of using the WL filtering (instead of the linear one) is maximum for one-dimensional constellation. Two-dimensional constellations (especially high-order ones) are often preferred to one-dimensional constellations (in presence of a linear receiver) in order to maximize the minimum distance between the constellation points [1]. However, WL linear filtering provides no performance advantage over linear one when the chosen constellation and the additive noise are circularly symmetric. For such a reason, we consider the optimization both over circularly symmetric and over rotationally variant constellations 
without any assumption about the circularity properties of the additive noise. In fact, the noncircularity of the constellation is introduced in order to exploit the presence of the WL receiver but it also provides a disadvantage in terms of the minimum distance between the constellation points.

When both the effects are accounted for, the optimum degree of noncircularity of the constellation becomes dependent on the specific channel impulse response. Therefore, we address the constellation design under the assumption that the channel state information (CSI) is available and we propose a CSI-dependent symbol mapping that optimizes the performance of the WL MMSE receiver. Symbol mapping is adapted by using a feedback channel (between the receiver and the transmitter) carrying information about the optimum constellation. Moreover, suboptimum strategies are proposed in order to reduce both the amount of information to be transmitted on the feedback channel and the computational complexity of the optimization procedure.

The paper is organized as follows. Section 2 introduces the system model, recalls the MMSE equalizer structure and analyzes how its performance depends on the amount of pseudocorrelation of the transmitted signal. Section 3 addresses the constellation design in the presence of the WL MMSE equalizer by generalizing the results in [2] to the case where the additive disturbance (noise plus interference) is rotationally variant. Section 4 reports the results of simulation experiments mainly aimed at showing the performance advantages provided by the constellation adaptation procedures. Finally, Section 5 provides the conclusions and the final remarks.

Notation 1. The following notations are adopted throughout the paper. $J$ is the imaginary unit, the superscripts $*, T$, and $H$ denote the complex-conjugate, the transpose and the Hermitian transpose, respectively, $E[\cdot]$ is the statistical expectation, $\delta_{k}$ is the Kronecker delta, $\mathbf{I}_{N}$ is the identity matrix of size $N, \mathbf{0}$ is the vector/matrix with all zero entries (the size is omitted for brevity), $a_{i}$ denotes the $i$ th entry of the vector $\mathbf{a}, a_{i k}$ denotes the $(i, k)$ entry of the matrix $\mathbf{A}, \mathbf{a}_{k}$ denotes the $k$ th column of $\mathbf{A}, \mathfrak{R}\{\cdot\}$ and $\mathfrak{I}\{\cdot\}$ are the real and the imaginary part, respectively, $\|\cdot\|_{p}$ denotes the $p$-norm with $\|\mathbf{a}\|_{-\infty} \triangleq \min _{i}\left|a_{i}\right|$, and, finally, $H(z) \triangleq \sum_{k=-\infty}^{+\infty} h_{k} z^{-k}$ is the $z$-transform of $h_{k}$.

\section{The FIR MMSE Equalizer}

In this section, we introduce the considered system model; then, we derive the WL MMSE feedforward-based equalizer and we study the variations of the achieved MMSE versus the pseudocorrelation of the transmitted signal. Such an analysis will be useful in Section 3 to address the constellation design for MMSE receivers.

2.1. System Model. Let us consider the following finiteimpulse-response (FIR) baseband-equivalent noisy commu- nication channel

$$
y_{k}=\sum_{\ell=0}^{v} h_{\ell} x_{k-\ell}+n_{k}
$$

where the transmitted symbols $x_{k}$ are independent identically distributed (i.i.d.) zero-mean random variables drawn from the complex-valued constellation $\mathbf{c} \in \mathbb{C}^{K}$ whose (finite) order $K$ determines the bit rate $\left(\log _{2} K\right.$ bits per symbol) of the uncoded system part. With no loss of generality, we assume that $E\left[x_{k} x_{k-\ell}^{*}\right]=\delta_{\ell}$ and $E\left[x_{k} x_{k-\ell}\right]=\beta \delta_{\ell}$, that is, the transmitted available power is unit, and that $x_{k}$ exhibits a possibly nonnull pseudocorrelation $\beta=E\left[\mathfrak{R}\left\{x_{k}\right\}^{2}\right]-$ $E\left[\Im\left\{x_{k}\right\}^{2}\right]+2 \jmath E\left[\Re\left\{x_{k}\right\} \mathfrak{I}\left\{x_{k}\right\}\right] \in \mathbb{C}$, such that $|\beta| \leq 1$ (if $|\beta| \leq 1$, then the correlation matrix of the $2 \times 1$ random vector $\left[\begin{array}{ll}x_{k} & x_{k}^{*}\end{array}\right]^{T}$ will be positive semidefinite); note that the noncircularity of $x_{k}$ consists in the difference between the power of the in-phase component and the quadrature one and in the correlation between them. Such assumption allows one to consider both the conventional circularly symmetric constellations $(\beta=0)$, such as $M$-PSK and square $M$-QAM with $M>2$, and the rotationally variant constellations, such as the well-known PAM $(\beta=1)$ and its rotated version (for which it exists $\theta$ such that $x_{k} e^{-j \theta}$ is real-valued and, consequently, $\beta=e^{j 2 \theta}$ ), non-square QAM (with $\beta=\mathfrak{R}(\beta) \neq 0$ since a different power is allocated to the in-phase and quadrature components). The timeinvariant FIR channel impulse response $h_{k}$ of memory $v$ is assumed to be known at the receiver side. Finally, the additive noise $n_{k}$, whose power $\sigma_{n}^{2}$ is assumed known at the receiver, is modeled as zero-mean complex-valued widesense stationary time-uncorrelated and independent of the useful signal. The additive disturbance $n_{k}$ is not assumed circularly symmetric because it may include the effects of one-dimensional cochannel interferences.

At the receiver side, the feedforward-based equalization is performed by processing the block of $N_{f}$ received samples $\mathbf{y}_{k} \triangleq\left[\begin{array}{llll}y_{k} & y_{k-1} & \ldots & y_{k-N_{f}+1}\end{array}\right]^{T}$ which, in a matrix notation, can be written as follows:

$$
\begin{aligned}
& \mathbf{y}_{k} \\
& =\left[\begin{array}{ccccccc}
h_{0} & h_{1} & \ldots & h_{\nu} & 0 & \ldots & 0 \\
0 & h_{0} & h_{1} & \ldots & h_{v} & 0 & \ldots \\
\vdots & & \ddots & & \ddots & & \vdots \\
0 & \ldots & 0 & h_{0} & h_{1} & \ldots & h_{\nu}
\end{array}\right]\left[\begin{array}{c}
x_{k} \\
x_{k-1} \\
\vdots \\
x_{k-\nu-N_{f}+1}
\end{array}\right]+\left[\begin{array}{c}
n_{k} \\
n_{k-1} \\
\vdots \\
n_{k-N_{f}+1}
\end{array}\right] \\
& =\mathbf{H x}_{k}+\mathbf{n}_{k} .
\end{aligned}
$$

According to the previous assumptions, the following correlation and pseudocorrelation matrices can be written as

$$
\begin{gathered}
\mathbf{R}_{x x} \triangleq E\left[\mathbf{x}_{k} \mathbf{x}_{k}^{H}\right]=\mathbf{I}_{N_{f}+v} \\
\mathbf{R}_{x x^{*}} \triangleq E\left[\mathbf{x}_{k} \mathbf{x}_{k}^{T}\right]=\beta \mathbf{I}_{N_{f}+v} \\
\mathbf{R}_{y y} \triangleq E\left[\mathbf{y}_{k} \mathbf{y}_{k}^{H}\right]=\mathbf{H} \mathbf{H}^{H}+\sigma_{n}^{2} \mathbf{I}_{N_{f}} \\
\mathbf{R}_{y y^{*}} \triangleq E\left[\mathbf{y}_{k} \mathbf{y}_{k}^{T}\right]=\beta \mathbf{H H}^{T}+\gamma \mathbf{I}_{N_{f}},
\end{gathered}
$$


where $\gamma \triangleq E\left[n_{k}^{2}\right]$ is the (possibly) nonnull noise pseudocorrelation (if $\gamma=0$, then the noise is circularly symmetric).

2.2. Feedforward-Based MMSE Equalizer. Since the transmitted sequence $x_{k}$ and consequently the received one $y_{k}$ in (1) can be rotationally variant, we adopt a widely linear receiver in order to exploit the statistical redundancy exhibited by the received signal. Note that such a choice improves the performance since the linear equalizers are a subset of the WL equalizers; their performances coincide only in the presence of circularly symmetric signals [10]. Therefore, we resort to the two FIR filters $\mathbf{w} \triangleq\left[\begin{array}{lllll}w_{0} & w_{1} & \cdots & w_{N_{f}-1}\end{array}\right]^{T}$ and $\mathbf{g} \triangleq$ $\left[\begin{array}{lllll}g_{0} & g_{1} & \cdots & g_{\mathrm{N}_{f}-1}\end{array}\right]^{T}$ that process the received vector $\mathbf{y}_{k}$ and its complex conjugate version $\mathbf{y}_{k}^{*}$, respectively. The optimum filters $\mathbf{w}^{(\mathrm{opt})}$ and $\mathbf{g}^{\text {(opt) }}$ minimizing the mean square error $E\left[\left|x_{k-\Delta}-\mathbf{w}^{H} \mathbf{y}_{k}-\mathbf{g}^{H} \mathbf{y}_{k}^{*}\right|^{2}\right]$ are given by $[16,18]$

$\mathbf{w}^{(\mathrm{opt})}=\left[\mathbf{R}_{y y}-\mathbf{R}_{y y *} \mathbf{R}_{y y}^{-*} \mathbf{R}_{y y^{*}}^{*}\right]^{-1}\left[\mathbf{h}_{\Delta+1}-\mathbf{R}_{y y *} \mathbf{R}_{y y}^{-*} \mathbf{h}_{\Delta+1}^{*} \beta^{*}\right]$,

$\mathbf{g}^{(\mathrm{opt})}=\left[\mathbf{R}_{y y}-\mathbf{R}_{y y^{*}} \mathbf{R}_{y y}^{-*} \mathbf{R}_{y y^{*}}^{*}\right]^{-*}\left[\mathbf{h}_{\Delta+1} \beta-\mathbf{R}_{y y *} \mathbf{R}_{y y}^{-*} \mathbf{h}_{\Delta+1}^{*}\right]^{*}$,

where $\mathbf{h}_{\Delta+1}$ denotes the $(\Delta+1)$ th column of $\mathbf{H}$ and the processing delay $0 \leq \Delta \leq N_{f}+v-1$ has to be chosen in order to optimize the performance. For notational simplicity, in (4) and (5) we have omitted the dependence of $\mathbf{w}^{\text {(opt) }}$ and $\mathbf{g}^{\text {(opt) }}$ on $\beta$. Let us point out that when $\beta=0$, that is, the transmitted symbols are drawn from a circularly symmetric constellation, $\mathbf{g}^{(\mathrm{opt})}=\mathbf{0}$ and, therefore, the $\mathrm{WL}$ MMSE equalizer degenerates into the conventional linear MMSE equalizer. Another special case is represented by the scenario where a real-valued constellation is adopted. In fact, since $\beta=1, \mathbf{g}^{\text {(opt) }}=\mathbf{w}^{\text {(opt) }^{*}}$ and the WL MMSE equalizer becomes $\mathfrak{R}\left\{2 \mathbf{w}^{(\mathrm{opt})^{H}} \mathbf{y}_{k}\right\}$, that is, it is implemented by extracting the in-phase component of the linear equalizer $\mathbf{w}^{\text {(opt) }}$, which does not coincide, however, with the linear MMSE equalizer.

Since the optimum equalizer and, hence, its performance depends on the pseudocorrelation $\beta$ of the transmitted signal, let us analyze the dependence on $\beta$ of the MMSE. To this end, denote with $e(\beta, \Delta) \triangleq x_{k-\Delta}-\mathbf{w}^{\left(\mathrm{optt}^{H}\right.} \mathbf{y}_{k}-\mathbf{g}^{(\mathrm{opt})^{H}} \mathbf{y}_{k}^{*}$ the error measured at the output of the WL MMSE equalizer for given values of $\beta$ and $\Delta$. It can be easily shown that

$$
\begin{aligned}
& \sigma_{e}(\beta, \Delta)^{2} \\
& \triangleq E\left[|e(\beta, \Delta)|^{2}\right] \\
& =1-\mathbf{w}^{\left(\mathrm{opt}^{H}\right.} \mathbf{h}_{\Delta+1}-\mathbf{g}^{(\mathrm{opt})^{H}} \mathbf{h}_{\Delta+1}^{*} \beta^{*},
\end{aligned}
$$

$$
\zeta(\beta, \Delta)
$$

$$
\begin{aligned}
\triangleq & \sigma_{e}(0, \Delta)^{2}-\sigma_{e}(\beta, \Delta)^{2} \\
= & {\left[\mathbf{h}_{\Delta+1} \beta-\mathbf{R}_{y y^{*}} \mathbf{R}_{y y}^{-*} \mathbf{h}_{\Delta+1}^{*}\right]^{T} } \\
& \times\left[\mathbf{R}_{y y}-\mathbf{R}_{y y^{*}} \mathbf{R}_{y y}^{-*} \mathbf{R}_{y y^{*}}^{*}\right]^{-*}\left[\mathbf{h}_{\Delta+1} \beta-\mathbf{R}_{y y^{*}} \mathbf{R}_{y y}^{-*} \mathbf{h}_{\Delta+1}^{*}\right]^{*} .
\end{aligned}
$$

Since $\sigma_{e}(0, \Delta)^{2}$ is the MMSE at the outputs of both the WL MMSE equalizer and the linear MMSE equalizer in the presence of a circularly symmetric constellation, $\zeta(\beta, \Delta)$ represents the MMSE gain achieved by properly choosing the pseudocorrelation $\beta$ of the transmitted constellation. When $\gamma=0$, that is, the noise is circularly symmetric, $\zeta(\beta, \Delta)$ depends on $|\beta|$ instead of $\beta$ and its (first) derivative with respect to $|\beta|$ can be written as

$$
\begin{aligned}
& \frac{\partial \zeta(\beta, \Delta)}{\partial|\beta|} \\
& =\frac{2}{|\beta|}\left[\mathbf{h}_{\Delta+1} \beta-\mathbf{R}_{y y^{*}} \mathbf{R}_{y y}^{-*} \mathbf{h}_{\Delta+1}^{*}\right]^{T}\left[\mathbf{R}_{y y}-\mathbf{R}_{y y^{*}} \mathbf{R}_{y y}^{-*} \mathbf{R}_{y y^{*}}^{*}\right]^{-*} \\
& \quad \times \mathbf{R}_{y y}^{*}\left[\mathbf{R}_{y y}-\mathbf{R}_{y y^{*}} \mathbf{R}_{y y}^{-*} \mathbf{R}_{y y^{*}}^{*}\right]^{-*}\left[\mathbf{h}_{\Delta+1} \beta-\mathbf{R}_{y y^{*}} \mathbf{R}_{y y}^{-*} \mathbf{h}_{\Delta+1}^{*}\right]^{*} .
\end{aligned}
$$

Since $\left[\mathbf{R}_{y y}-\mathbf{R}_{y y *} \mathbf{R}_{y y}^{-*} \mathbf{R}_{y y^{*}}^{*}\right]^{-*}$ and $\mathbf{R}_{y y}$ are positive semidefinite, one has $(\partial \zeta(\beta, \Delta) / \partial|\beta|) \geq 0$ and, hence, increasing the degree of noncircularity of the transmitted signal improves the MMSE. For such a reason, the use of a real-valued transmitted sequence together with a WL MMSE equalizer corresponds to the optimum choice as far as the MMSE is adopted as the performance measure. On the other hand, when $\gamma \neq 0$, the variations of $\zeta(\beta, \Delta)$ with respect to $\beta$ depend on the specific values of the channel impulse response and the noise statistics.

\section{Constellation Design}

The present section addresses the design of the $K$-order constellation with $K$ fixed (under the assumption that the WL MMSE equalizer is used) and it is organized as follows. In Section 3.1, we address the optimum constellation design for the WL MMSE receiver by extending the results of [2] to the case of additive rotationally variant disturbance. In Section 3.2, we propose a suboptimum strategy based on the rhombic transformation of a given constellation. Such a strategy allows one to reduce both the computational complexity of the optimization procedure and the amount of information required at the transmitting side in order to adapt the constellation.

The results in the previous section allow one to state that, by using a real-valued constellation $(\beta=1)$ instead of a complex-valued nonredundant $(\beta=0)$ one, a performance gain can be achieved in terms of the MMSE at the equalizer output. On the other hand, not always an MSE gain provided by the WL equalizer leads to a SER gain [19]. In fact, for a fixed expended average energy per bit, the reduction of the minimum distance between the constellation points, due to the adoption of one-dimensional constellations rather than two-dimensional ones (e.g., when we adopt the $K$ PAM rather than the $K$-QAM) leads to a potential increase in the SER. Therefore, we address the constellation design minimizing the SER at the WL MMSE equalizer output by accounting for its rotationally variant properties.

In the literature (e.g., $[2,20]$ ), most of the constellations employed by the transmission stage are circularly symmetric 


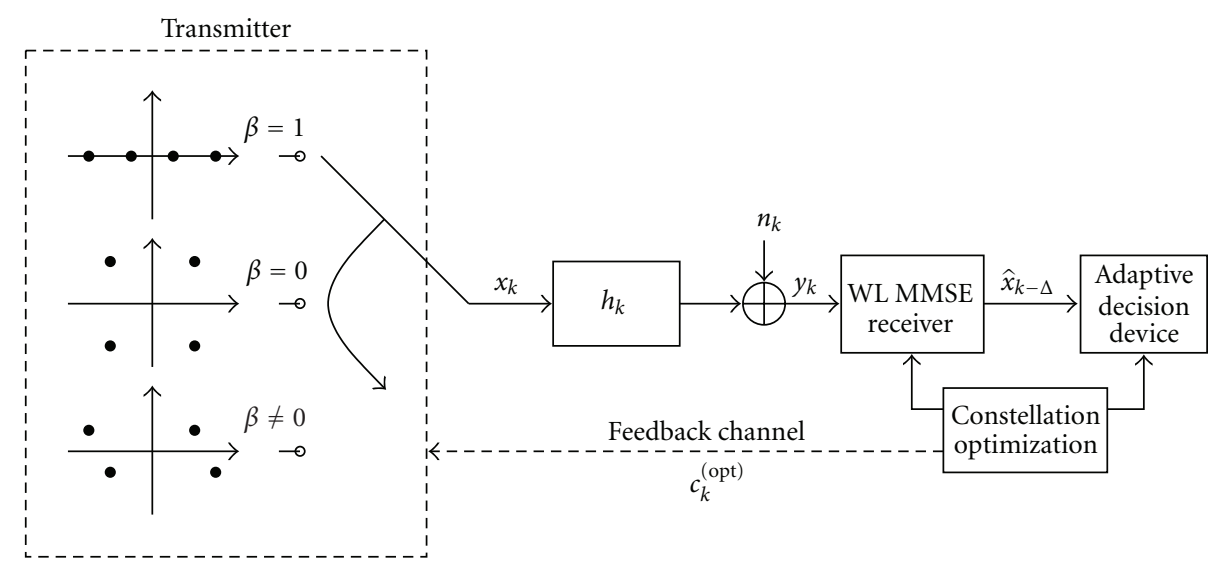

FIgURE 1: Transceiver structure.

$(\beta=0)$, while statistically redundant constellations are confined to the real-valued ones. Moreover, in [2], with reference to the transmission over a time nondispersive channel $\left(h_{k}=\delta_{k}\right)$ affected by circularly symmetric noise, a procedure for constellation optimization has been proposed, showing also that, for large signal-to-noise ratios (SNR), the performance of the conventional QAM maximum-likelihood (ML) receiver is invariant with respect to rhombic transformations of the complex plane. However, it is important to point out that a rhombic transformation of a circular constellation makes it rotationally variant and, for some values of $K$ (e.g., $K=8$ ), the procedure in [2] provides a rotationally variant constellation. On the other hand, the WL equalizer is equivalent to the linear equalizer over the nondispersive channel considered in [2] and, therefore, optimizing the circularity degree of the constellation does not provide any performance advantage. On the other hand, when a time-dispersive channel is considered, the WL MMSE equalizer is sensitive to the rotationally variant properties of the transmitted signal and, therefore, we propose a transceiver structure (see Figure 1) where (i) the transmitter can switch between the available constellations of order $K$; (ii) the WL MMSE receiver accounts for the CSI and informs the transmitter, by means of a feedback channel, about which constellation has to be adopted to minimize the SER.

The use of a feedback channel in order to improve the bit-rate could also be exploited for choosing the constellation size rather than its circularity degree when the signal-tonoise ratio of each channel realization is not previously known. For example, the problem of the constellation choice has been addressed in $[21,22]$ with reference to the discrete multitone (DMT) transceiver and to multiple-input multiple-output transceiver, respectively. The two parameters of the constellations (size and circularity-degree) could also be jointly optimized by generalizing the procedures here proposed.

3.1. Constellation Optimization in the Presence of Gaussian Rotationally Variant Noise. In order to optimize over the constellation choice we need to first derive a performance analysis of the considered equalizer. Approximated evaluations of the performance of the WL receiver are available in [11] for a QAM constellation and in [3] for a PAM constellation in the presence of a PAM cochannel interference. Moreover, such performance analysis is generalized in [9] for IIR WL filters. Here, we derive an approximation of the equalizer performance suited for successive optimization over transmitter constellation.

With no loss of generality, assume that $\Delta=0$ and rewrite the output of the FIR equalizer as follows:

$$
\begin{aligned}
z_{k}(\beta) & =\mathbf{w}^{(\mathrm{opt})^{H}} \mathbf{y}_{k}+\mathbf{g}^{(\mathrm{opt})^{H}} \mathbf{y}_{k}^{*} \\
& =x_{k}(\beta)+e_{k}(\beta)
\end{aligned}
$$

where $x_{k}(\beta)$ is the transmitted symbol drawn from the complex-valued constellation $\mathbf{c} \triangleq\left[\begin{array}{llll}c_{1} & c_{2} & \cdots & c_{K}\end{array}\right]^{T}$ with $E\left[\left|x_{k}(\beta)\right|^{2}\right]=1$ and $E\left[x_{k}(\beta)^{2}\right]=\beta$, and $e_{k}(\beta)$ is the residual disturbance that includes the intersymbol interference and the noise terms after the WL equalizer filtering. The circularly symmetric model for the additive disturbance is inadequate since the output of a WL filter is, in general, rotationally variant. Therefore, we model $e_{k}(\beta)$ as rotationally variant, that is, $E\left[\Re\left\{e_{k}(\beta)\right\}^{2}\right] \triangleq \sigma_{e, R}(\beta)^{2}, E\left[\mathfrak{I}\left\{e_{k}(\beta)\right\}^{2}\right] \triangleq \sigma_{e, I}(\beta)^{2}=$ $\sigma_{e}(\beta)^{2}-\sigma_{e, R}(\beta)^{2}$, and $E\left[\Re\left\{e_{k}(\beta)\right\} \mathfrak{I}\left\{e_{k}(\beta)\right\}\right]=\sigma_{e, R I}(\beta)$. Moreover, in order to make the constellation design analytically tractable, we approximate $e_{k}(\beta)$ as Gaussian. For the sake of clarity, let us note that, if symbols $x_{k}(\beta)$ and noise are circularly symmetric $(\beta=\gamma=0)$, then the additive disturbance $e_{k}(0)$ and the equalizer output $z_{k}(0)$ will be circularly symmetric too; on the other hand, if $x_{k}(\beta)$ is rotationally variant, then $z_{k}(\beta)$ will be rotationally variant too, but nothing can be stated about the circularity properties of $e_{k}(\beta)$ also when $\gamma=0$.

The sample $z_{k}(\beta)$ is the input of the decision device which performs the symbol-by-symbol ML detection of the transmitted symbol. By defining the following eigenvalue 
decomposition (the dependence on $\beta$ at the right-hand-side is omitted for simplicity):

$$
\begin{aligned}
& {\left[\begin{array}{ll}
\sigma_{e, R}(\beta)^{2} & \sigma_{e, R I}(\beta) \\
\sigma_{e, R I}(\beta) & \sigma_{e, I}(\beta)^{2}
\end{array}\right]} \\
& \triangleq \underbrace{\left[\begin{array}{ll}
v_{11} & v_{12} \\
v_{12} & v_{22}
\end{array}\right]}_{\mathbf{V}} \underbrace{\left[\begin{array}{cc}
s_{1} & 0 \\
0 & s_{2}
\end{array}\right]}_{\mathbf{S}} \underbrace{\left[\begin{array}{ll}
v_{11} & v_{12} \\
v_{12} & v_{22}
\end{array}\right]^{T}}_{\mathbf{V}^{T}} \quad\left(s_{1} \geq s_{2} \geq 0\right),
\end{aligned}
$$

with $\mathbf{V}$ being the eigenvector matrix and $\mathbf{S}$ having on the diagonal the eigenvalues, it can be verified that the pair-wise error probability $P\left(c_{i} \rightarrow c_{\ell}\right)$ [20], that is, the probability of transmitting $c_{i}$ and deciding (at the receiver) in favor of $c_{\ell}$ when the transmission system uses only $c_{i}$ and $c_{\ell}$, is given by

$$
\begin{aligned}
& P\left(c_{i} \longrightarrow c_{\ell} ; \beta\right) \\
& =\frac{1}{2} \operatorname{erfc}\left(\frac{1}{2 \sqrt{2}} \sqrt{\mathfrak{e}+\psi_{R I}\left(c_{i, R}-c_{\ell, R}\right)\left(c_{i, I}-c_{\ell, I}\right)}\right),
\end{aligned}
$$

where $\mathfrak{e}$ denotes $\left(c_{i, R}-c_{\ell, R}\right)^{2} / \psi_{R}(\beta)+\left(c_{i, I}-c_{\ell, I}\right)^{2} / \psi_{I}(\beta)$, where $c_{k, R} \triangleq \mathfrak{R}\left\{c_{k}\right\}$ and $c_{k, I} \triangleq \mathfrak{I}\left\{c_{k}\right\}$, and, for $s_{1} \neq 0$ and $s_{2} \neq 0$,

$$
\begin{gathered}
\psi_{R}(\beta) \triangleq\left(\frac{v_{11}^{2}}{s_{1}}+\frac{v_{12}^{2}}{s_{2}}\right)^{-1}, \\
\psi_{I}(\beta) \triangleq\left(\frac{v_{12}^{2}}{s_{1}}+\frac{v_{22}^{2}}{s_{2}}\right)^{-1}, \\
\psi_{R I}(\beta) \triangleq 2\left(\frac{v_{11}}{s_{1}}+\frac{v_{22}}{s_{2}}\right) v_{12} .
\end{gathered}
$$

When $s_{2}=0, \psi_{R}(\beta) \triangleq s_{1} / v_{11}^{2}$ and analogously for $\psi_{I}(\beta)$ and $\psi_{R I}(\beta)$. By utilizing (11), assuming that the symbols $c_{k}$ are equally probable, and resorting to both the union bound and Chernoff bound techniques, the $\operatorname{SER} P_{e}^{(\text {true })}(\mathbf{c})$ is upperbounded as follows:

$$
\begin{aligned}
P_{e}^{(\text {true })}(\mathbf{c}) \leq P_{e}(\mathbf{c} ; \beta) & \\
\triangleq \frac{1}{K} \sum_{i=1}^{K} \sum_{\ell \neq i} \exp \left\{-\frac{1}{8}[\right. & \frac{\left(c_{i, R}-c_{\ell, R}\right)^{2}}{\psi_{R}(\beta)}+\frac{\left(c_{i, I}-c_{\ell, I}\right)^{2}}{\psi_{I}(\beta)} \\
& \left.\left.+\psi_{R I}\left(c_{i, R}-c_{\ell, R}\right)\left(c_{i, I}-c_{\ell, I}\right)\right]\right\}
\end{aligned}
$$

and, therefore, the optimum constellation can be approximated with the solution $\mathbf{c}^{\text {(opt) }}$ of the following problem:

$$
\begin{gathered}
\mathbf{c}^{(\text {opt })}=\arg \min _{\mathbf{c} \in \mathbb{C}^{K}, \beta \in \mathbb{C}} P_{e}(\mathbf{c} ; \beta), \\
\frac{1}{K} \sum_{i=1}^{K}\left|c_{i}\right|^{2}=1 \\
\frac{1}{K} \sum_{i=1}^{K} c_{i}^{2}=\beta \\
|\beta| \leq 1
\end{gathered}
$$

Unfortunately, it is difficult to find the closed-form expression of the solution of such an optimization problem. For such a reason, we propose to find a local solution by means of numerical algorithms (e.g., a projected gradient method). To this aim, we can exploit the gradient of $P_{e}(\mathbf{c} ; \beta)$ with respect to $\mathbf{c}$, while we resort to numerical approximation of the gradient with respect to $\beta$ since it is difficult to obtain its analytical expression.

Before proceeding, let us discuss the property of the locally optimum constellation for a fixed $\beta$. The $k$ th component of the gradient of $P_{e}(\mathbf{c} ; \beta)$ is given by

$$
\begin{aligned}
& \frac{\partial P_{e}(\mathbf{c} ; \beta)}{\partial c_{k}} \\
& =-\frac{1}{2 K} \sum_{\ell \neq k} \exp \left\{-\frac{1}{8}\left[\frac{\left(c_{k, R}-c_{\ell, R}\right)^{2}}{\psi_{R}(\beta)}+\frac{\left(c_{k, I}-c_{\ell, I}\right)^{2}}{\psi_{I}(\beta)}\right.\right. \\
& \left.\left.+\psi_{R I}(\beta)\left(c_{k, R}-c_{\ell, R}\right)\left(c_{k, I}-c_{\ell, I}\right)\right]\right\} \\
& \times\left[\frac{c_{k, R}-c_{\ell, R}}{\psi_{R}(\beta)}+j \frac{c_{k, I}-c_{\ell, I}}{\psi_{I}(\beta)}+j \frac{\psi_{R I}(\beta)}{2}\right. \\
& \left.\times\left(\left(c_{k, R}-c_{\ell, R}\right)-\jmath\left(c_{k, I}-c_{\ell, I}\right)\right)\right] .
\end{aligned}
$$

By zeroing the gradient of the Lagrangian

$$
\begin{aligned}
& F\left(\mathbf{c}, \beta, \lambda_{1}, \lambda_{2}, \lambda_{3}\right) \\
& \triangleq P_{e}(\mathbf{c} ; \beta)+\lambda_{1}\left(\frac{1}{K} \sum_{k=1}^{K}\left|c_{k}\right|^{2}-1\right) \\
& \quad+\lambda_{2}\left(\frac{1}{K} \sum_{k=1}^{K}\left(c_{k, R}^{2}-c_{k, I}^{2}\right)-\Re\{\beta\}\right) \\
& \quad+\lambda_{3}\left(\frac{1}{K} \sum_{k=1}^{K} c_{k, R} c_{k, I}-\mathfrak{I}\{\beta\}\right)
\end{aligned}
$$

one has that the locally optimum $\mathrm{c}$ satisfies the following equation:

$$
\begin{aligned}
& \frac{1}{2} \sum_{\ell \neq k} \xi(k, \ell)[ \frac{c_{k, R}-c_{\ell, R}}{\psi_{R}(\beta)}+\jmath \frac{c_{k, I}-c_{\ell, I}}{\psi_{I}(\beta)} \\
&\left.+\jmath \frac{\psi_{R I}(\beta)}{2}\left(\left(c_{k, R}-c_{\ell, R}\right)-\jmath\left(c_{k, I}-c_{\ell, I}\right)\right)\right] \\
&=2 \lambda_{1} c_{k}+2\left(\lambda_{2}+\jmath \lambda_{3}\right) c_{k}^{*}
\end{aligned}
$$

with

$\xi(k, \ell)$

$$
\begin{aligned}
\triangleq \exp \left\{-\frac{1}{8}\left[\frac{\left(c_{k, R}-c_{\ell, R}\right)^{2}}{\psi_{R}(\beta)}+\frac{\left(c_{k, I}-c_{\ell, I}\right)^{2}}{\psi_{I}(\beta)}\right.\right. \\
\left.\left.+\psi_{R I}(\beta)\left(c_{k, R}-c_{\ell, R}\right)\left(c_{k, I}-c_{\ell, I}\right)\right]\right\} .
\end{aligned}
$$


Condition (17) generalizes the result of [2] to the case of $e_{k}$ rotationally variant (i.e., $\sigma_{e, R}(\beta)^{2} \neq \sigma_{e, I}(\beta)^{2}$ or $\sigma_{e, R I}(\beta) \neq 0$ ) and with a constrained pseudocorrelation. $\left(\partial f(\mathbf{c}) / \partial c_{k}=\right.$ $\partial f(\mathbf{c}) / \partial c_{k, R}+j\left(\partial f(\mathbf{c}) / \partial c_{k, I}\right)$.) In fact, (17) with $\lambda_{2}=\lambda_{3}=0$ (i.e., no constraint is imposed on the pseudocorrelation) requires that $c_{k}$ is proportional to the weighted sum (with weights $\left.\xi(k, \ell) / \psi_{R}(\beta)\right)$ of $c_{k}-c_{\ell}, \forall \ell \neq k$, as found in [2]. For the sake of clarity, let us note that the procedure proposed in [2] does not allow one to exploit the potential advantage of a rotationally variant constellation when the WL MMSE receiver is employed. For example, when a linear MMSE equalizer is employed for $K=4$ in high signal-to-noise ratio, the minimum of the SER is equivalently achieved [2] by both the conventional 4-QAM constellation and the rhombic constellations with the same perimeter, that is, the perimeter of the largest convex polygon consisting of the lines $c_{k}-c_{\ell}$ (see [1] for further details). On the other hand, when a WL MMSE equalizer is employed, a rhombic constellation, which is rotationally variant, is not equivalent to the conventional 4-QAM since the achieved MMSE is dependent on $\beta$ as shown in (8).

3.2. A Suboptimum Procedure Based on Rhombic Transformations. In this section, we propose a suboptimum constellation-design procedure for the WL MMSE equalizer. The method is based on the exploitation of a rhombic transformation that operates on a circularly symmetric constellation making it rotationally variant. Such a transformation depends on two parameters and allows one to control the pseudocorrelation $\beta$ of the obtained constellation; consequently, the optimization procedure is simplified since the SER in (13) is a function of only two parameters, instead of $K$ parameters.

Assume that $\mathbf{c}=\left[\begin{array}{llll}c_{1} & c_{2} & \cdots & c_{K}\end{array}\right]^{T}$ is a unit-power circularly-symmetric complex-valued constellation and define the complex-valued constellation $\tilde{\mathbf{c}}=\left[\begin{array}{lll}\tilde{c}_{1} & \tilde{c}_{2}\end{array} \ldots\right.$ $\left.\tilde{c}_{K}\right]^{T}$ as follows:

$$
\begin{aligned}
& {\left[\begin{array}{c}
\mathfrak{R}\left\{\tilde{c}_{k}\right\} \\
\mathfrak{I}\left\{\tilde{c}_{k}\right\}
\end{array}\right]} \\
& =\frac{1}{\sqrt{1+\alpha^{2}}}\left[\begin{array}{cc}
(1+\alpha) \cos (\theta / 2) & -(1+\alpha) \sin \left(\frac{\theta}{2}\right) \\
-(1-\alpha) \sin (\theta / 2) & (1-\alpha) \cos \left(\frac{\theta}{2}\right)
\end{array}\right]\left[\begin{array}{c}
\mathfrak{R}\left\{c_{k}\right\} \\
\Im\left\{c_{k}\right\}
\end{array}\right]
\end{aligned}
$$

or, more compactly (the compact expression is introduced for notation simplicity whereas the matrix form is utilized to understand the physical meaning),

$$
\begin{gathered}
\tilde{c}_{k}=\underbrace{\frac{1}{\sqrt{1+\alpha^{2}}}\left[\cos \left(\frac{\theta}{2}\right)+\jmath \alpha \sin \left(\frac{\theta}{2}\right)\right]}_{\triangleq_{\mu(\alpha, \theta)}} c_{k} \\
+\underbrace{\frac{1}{\sqrt{1+\alpha^{2}}}\left[\alpha \cos \left(\frac{\theta}{2}\right)-\jmath \sin \left(\frac{\theta}{2}\right)\right]}_{\triangleq_{\kappa(\alpha, \theta)}} c_{k}^{*},
\end{gathered}
$$

with $-1 \leq \alpha \leq 1$ and $-\pi / 2 \leq \theta \leq \pi / 2$. When $\alpha>0(\alpha<0)$, $\tilde{c}_{k}$ is stretched along the in-phase (quadrature) component and it becomes one-dimensional for $\alpha= \pm 1$; when $\theta \neq 0$, a correlation between $\mathfrak{R}\left\{\widetilde{c}_{k}\right\}$ and $\mathfrak{I}\left\{\widetilde{c}_{k}\right\}$ is introduced and for $\theta= \pm \pi / 2$, even if it is two-dimensional, $\tilde{c}_{k}$ can be reduced to a one-dimensional constellation by a simple rotation. For symmetry, in the following we consider only the positive values of $\alpha$ and $\theta$. It is easily verified that, if $x_{k}$ is drawn from $\tilde{\mathbf{c}}$, then

$$
E\left[\left|x_{k}\right|^{2}\right]=1, \quad \beta=2 \mu(\alpha, \theta) \kappa(\alpha, \theta) .
$$

The method proposed here assumes that the information-bearing symbol sequence, say $s_{k}$, is drawn from a fixed constellation c (e.g., the optimum constellation provided by [2]) whereas the possibly rotationally variant channel input $x_{k}$ is obtained by resorting to the zeromemory precoding defined by the rhombic transformation (19). Clearly, such a strategy is suboptimum since it assumes that the channel input can be drawn from only those constellations $\tilde{\mathbf{c}}$ resulting from a rhombic transformation of the chosen $\mathbf{c}$. However, the main advantages of such a method in comparison with the optimum one are

(1) the huge reduction of the computational complexity of the constellation optimization procedure when $K \gg 1$; in fact, the SER becomes a function of only two variables $(\alpha$ and $\theta$ ), regardless of the constellation order $K$;

(2) the reduced implementation complexity of the transmitter stage; in fact, the symbol-mapping is implemented by means of the linear transformation (19);

(3) the decrease of the information amount to be transmitted on the feedback channel; in fact, only the values of two parameters (instead of $K$ ) have to be sent to the transmitter.

According to such a choice, the constellation optimization is carried out by solving the minimization problem

$$
\left(\alpha^{(\mathrm{opt})}, \theta^{\text {(opt) }}\right)=\arg \min _{\alpha, \theta} P_{e}(\alpha, \theta),
$$

with

$$
\begin{aligned}
& P_{e}(\alpha, \theta) \\
& =\frac{1}{K} \sum_{i=1 \ell \neq i}^{K} \sum_{\ell} \exp \left\{-\frac{1}{8\left(1+\alpha^{2}\right)}\right. \\
& \times\left[\frac{(1+\alpha)^{2}}{\psi_{R}(\alpha, \theta)}\left(\mathfrak{d}-\sin \left(\frac{\theta}{2}\right)\left(c_{i, I}-c_{\ell, I}\right)\right)^{2}\right. \\
& \left.\left.+\frac{(1-\alpha)^{2}}{\psi_{I}(\alpha, \theta)}(\mathfrak{f})^{2}-\psi_{R I}(\alpha, \theta) \frac{1-\alpha^{2}}{1+\alpha^{2}} \mathfrak{g}\right]\right\},
\end{aligned}
$$

where $\mathfrak{d}$ denotes $\cos (\theta / 2)\left(c_{i, R}-c_{\ell, R}\right)$, $\mathfrak{f}$ denotes $\sin (\theta /$ 2) $\left(c_{i, R}-c_{\ell, R}\right)-\cos (\theta / 2)\left(c_{i, I}-c_{\ell, I}\right)$, and $\mathfrak{g}$ denotes ((1/ 2) $\left.\sin (\theta)\left(\left(c_{i, R}-c_{\ell, R}\right)^{2}+\left(c_{i, I}-c_{\ell, I}\right)^{2}\right)-\left(c_{i, R}-c_{\ell, R}\right)\left(c_{i, I}-c_{\ell, I}\right)\right)$, 
and where (23) follows from (13) and (19), and the dependence of the disturbance parameters on $\beta$ has been replaced by the dependence on $\alpha$ and $\theta$. Since finding the closed-form expression of $\alpha^{(\mathrm{opt})}$ and $\theta^{(\mathrm{opt})}$ is a difficult problem, here we propose to approximate $P_{e}(\alpha, \theta)$ with a function, say $P_{e}^{(\text {low })}(\alpha, \theta)$, whose minimization can be carried out by evaluating it only over a very limited set of points. In the sequel, such an approximation is derived for a 4QAM constellation $c_{k}=1 / \sqrt{2}( \pm 1 \pm J)$, though it can be analogously determined for denser constellations.

First, we approximate the cost function (23) by assuming that the components of the residual disturbance are uncorrelated, that is, $\psi_{R I}(\alpha, \theta)=0$. By means of some tedious but simple algebra operations, it can be shown that $P_{e}(\alpha, \theta)$ is lower bounded by

$$
P_{e}(\alpha, \theta) P_{e}^{(\text {low })}(\alpha, \theta) \triangleq \exp \left\{-\frac{1}{4}\|\Sigma(\alpha, \theta)\|_{-\infty} \cdot d_{\min }(\alpha, \theta)\right\},
$$

where

$$
\begin{gathered}
\boldsymbol{\Sigma}(\alpha, \theta) \triangleq\left[\begin{array}{l}
\psi_{R}(\alpha, \theta)^{-1} \\
\psi_{I}(\alpha, \theta)^{-1}
\end{array}\right] \\
d_{\min }(\alpha, \theta) \triangleq \min _{\ell \in\{0, \pm 1\}}\left\|\mathbf{d}_{\ell}(\alpha, \theta)\right\|_{1} \\
\mathbf{d}_{\ell}(\alpha, \beta) \triangleq \frac{1}{1+\alpha^{2}}\left[\begin{array}{c}
(1+\alpha)^{2}[\mathfrak{a}]^{2} \\
(1-\alpha)^{2}[\mathfrak{b}]^{2}
\end{array}\right],
\end{gathered}
$$

where $\mathfrak{a}$ denotes $\left(\delta_{\ell}+\delta_{\ell-1}\right) \cos (\theta / 2)-\left(\delta_{\ell}+\delta_{\ell+1}\right) \sin (\theta / 2)$ and $\mathfrak{b}$ denotes $\left(\delta_{\ell}+\delta_{\ell-1}\right) \sin (\theta / 2)-\left(\delta_{\ell}+\delta_{\ell+1}\right) \cos (\theta / 2)$. Since the right-hand side of $(24)$ is minimized by large values of $d_{\min }(\bar{\alpha}, \theta)$, we propose to approximate the solution of $(22)$ with the following one:

$$
\begin{gathered}
\left(\hat{\alpha}^{(\mathrm{opt})}, \hat{\theta}^{(\mathrm{opt})}\right)=\arg \min _{(\alpha, \theta) \in \mathcal{X}} P_{e}^{(\mathrm{low})}(\alpha, \theta), \\
X \triangleq\left\{(\alpha, \theta): 2 \sin (\theta)-\frac{2 \alpha}{1+\alpha^{2}} \cos (\theta)=1\right\},
\end{gathered}
$$

where $\mathcal{X}$ is the $(\alpha, \theta)$-curve corresponding to the maximum value of $d_{\min }(\bar{\alpha}, \theta)$ for a fixed $\alpha=\bar{\alpha}$ (or, equivalently, to the maximum value of $d_{\min }(\alpha, \bar{\theta})$ for a fixed $\left.\theta=\bar{\theta}\right)$. Of course, the restriction to $\mathcal{X}$ leads to a significant decrease in the computational complexity. Let us point out that, interestingly, such a restricted optimization procedure accounts for the possible transmission of the conventional 4-PAM: in fact, it can be easily verified that when $\left(\alpha_{\text {PAM }}, \theta_{\text {PAM }}\right) \triangleq$ $\left(1, \tan ^{-1}(4 / 3)\right) \in \mathcal{X}, \widetilde{c}_{k}=\{ \pm(1 / \sqrt{5}), \pm(3 / \sqrt{5})\}$.

This also suggests an extreme simplification obtained by choosing just between the 4-PAM and 4-QAM constellation (two-choice procedure), that is, one can resort to an architecture that switches between the 4-QAM and the 4-PAM constellations according to the following rule:

$$
P_{e}^{(\text {low })}\left(\alpha_{\mathrm{PAM}}, \theta_{\mathrm{PAM}}\right) \underset{\text { PAM }}{\stackrel{\mathrm{QAM}}{\gtrless}} P_{e}^{(\text {low })}(0,0) .
$$

Three remarks about the suboptimum procedure (26) follow.
Remark 1. The results carried out here with reference to the 4-QAM constellation can be easily generalized to higher-order constellations. More specifically, the SERbound approximations (analogous to the one in (24)) can be obtained by assuming that the inner summation in (23) is restricted to those constellation points closest to the $k$ th one. Moreover, it can be shown that the conventional square $K$-QAM constellations (with $K=16,64,128$ ) can be transformed by (19) into the conventional uniform $K$ PAM. Note, however, that such a property is not satisfied by the constellations of any order; for example, as also shown in Section 4, when using the rectangular 8-QAM (see Figure $2(\mathrm{~g})$ ) the rhombic transformation allows one to obtain the nonuniform 8-PAM reported in Figure 2(i).

Remark 2. The optimum transmission strategy proposed here requires that the receiver sends on the feedback channel the whole optimum constellation. If the suboptimum procedure is used, the transmitter architecture can be simplified. In fact, a unique symbol mapper for the alphabet c is needed and the constellation is adapted by adjusting the zero-memory WL filter (19). Unfortunately, the main disadvantage in terms of the computational complexity of the receiver remains the adaptation of the decision mechanism for the constellation $\tilde{\mathbf{c}}$.

Remark 3. When the proposed suboptimum strategy is used, the channel input $x_{k}$ is obtained by performing a zeromemory WL filtering of the information-bearing sequence $s_{k}$. For such a reason, it is reasonable to consider an alternative receiver structure that performs the WL MMSE equalization of the received signal in order to estimate $s_{k-\Delta}$, instead of $x_{k-\Delta}$. After some matrix manipulations, it can be verified that such WL MMSE equalizer is the cascade of the WL MMSE equalizer in (4) and (5) and the WL zeromemory filter performing the inverse of the transformation (19) (note that (19) is not invertible for every value of $\alpha$ and $\theta$, e.g., when a real-valued constellation is adopted $(\alpha=1)$, however, in such a case, an ad hoc inverse transformation can be easily defined). This allows one to use a unique symbol de-mapper and the standard decision mechanism for the constellation c. The MMSE achieved by such a structure is

$$
\begin{aligned}
& E\left[\left|e_{s}\right|^{2}\right] \\
& \triangleq E\left[\left|s_{k-\Delta}-\mathbf{w}_{s}^{\left(\text {opt }^{H}\right.} \mathbf{y}_{k}-\mathbf{g}_{s}^{\left(\text {opt }^{H}\right.} \mathbf{y}_{k}^{*}\right|^{2}\right] \\
& =1+\frac{1}{|\mu|^{2}-|\kappa|^{2}} \\
& \quad \times\left[\left(\sigma_{e}(\beta)^{2}-1\right)+4|\mu|^{2}|\kappa|^{2}-\mathfrak{R}\left\{\beta^{*} E\left[e(\beta)^{2}\right]\right\}\right] .
\end{aligned}
$$

It can be easily shown that $(\mathrm{a})$ if $\sigma_{e}(\beta)^{2} \rightarrow 0$, then $E\left[\left|e_{s}\right|^{2}\right] \rightarrow$ 0 , unless $|\mu|^{2}=|\kappa|^{2}$, and (b) $E\left[\left|e_{s}\right|^{2}\right] \geq \sigma_{e}(\beta)^{2}$ since $|\mu|^{2}-|\kappa|^{2} \leq 1$. Such results show that the minimumdistance decision based on the WL MMSE estimation of 


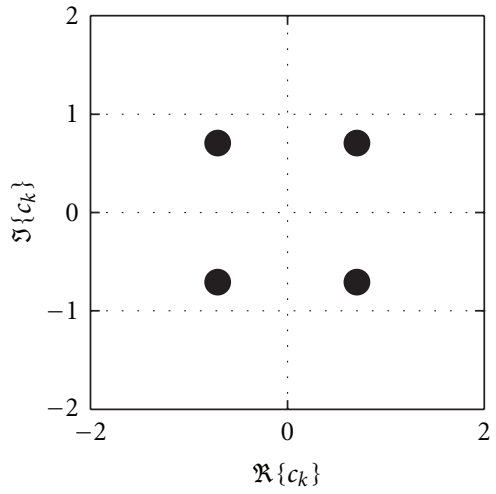

(a)

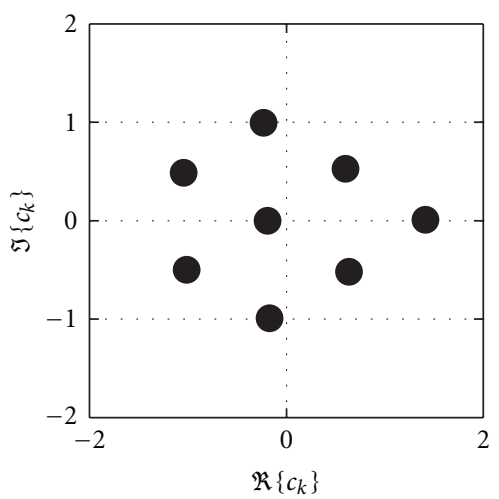

(d)

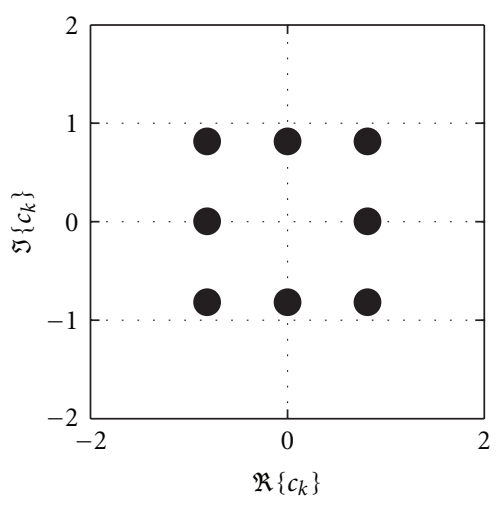

(g)

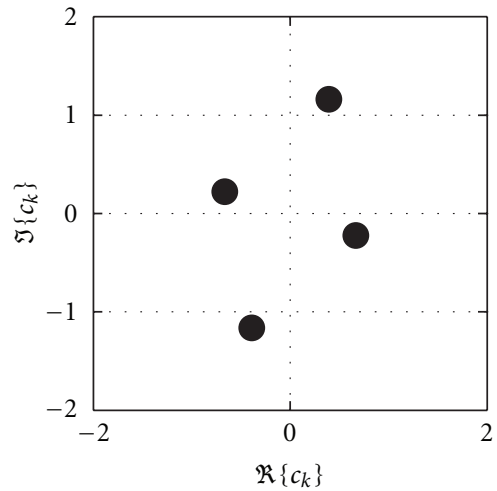

(b)

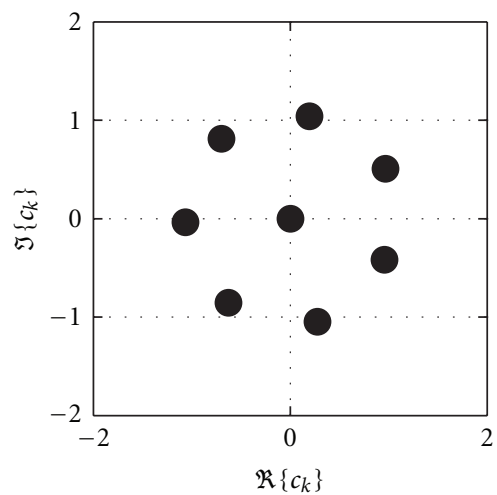

(e)

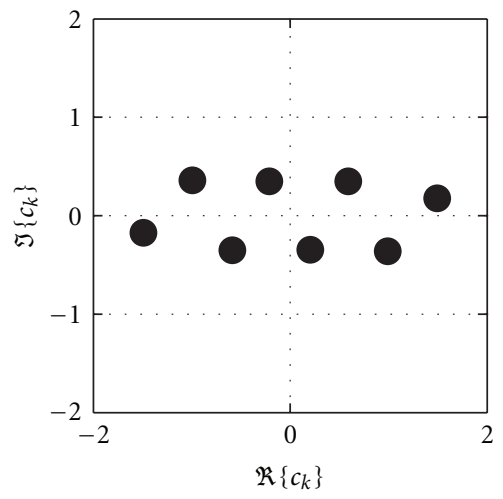

(h)

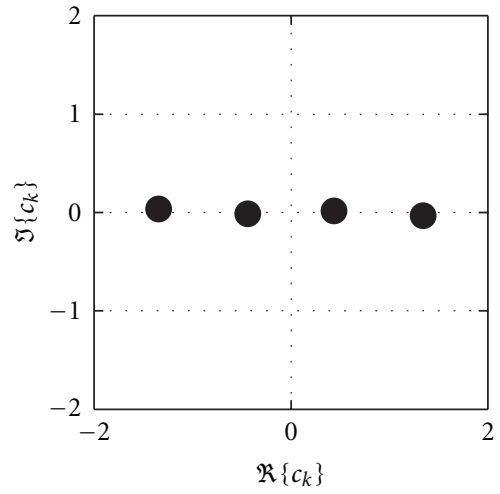

(c)

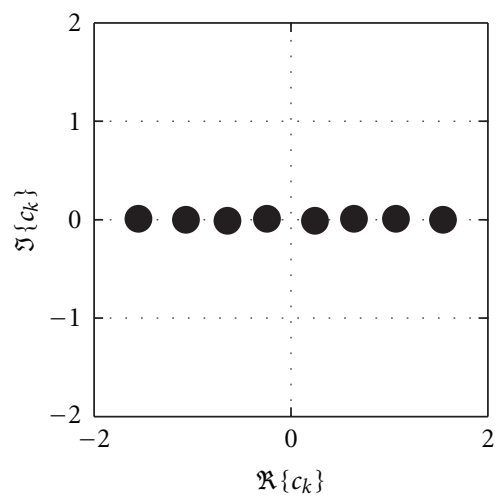

(f)

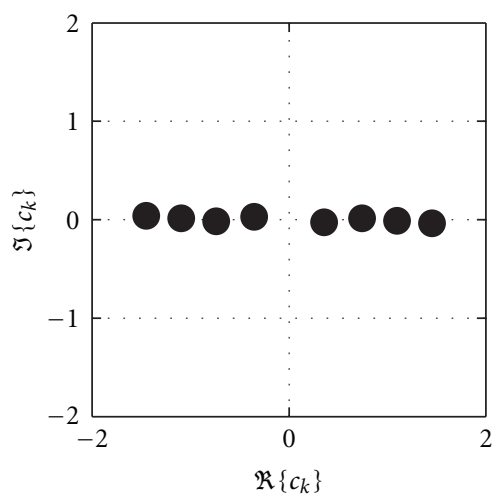

(i)

FIGURE 2: Optimum constellations for $K=4$ and $K=8$. (a) QPSK, (b) Rhombic QPSK, (c) 4-PAM, (d) Foschini and All 8-QAM, (e) "1-7" 8-QAM, (f) 8-PAM, (g) rectangular 8-QAM, (h) noncircular 8-QAM, and (i) nonuniform 8-QAM.

$x_{k-\Delta}$ outperforms the (computationally simpler) minimumdistance decision based on the WL MMSE estimation of $s_{k-\Delta}$.

\section{Numerical Results}

In this section, we present the results of simulation experiments aimed at assessing the performance improvements achievable by the proposed constellation-optimization pro- cedures. In all the experiments, we assume that (1) the noise sequence at the output of the channel is zero-mean white Gaussian complex-valued circularly symmetric with variance $\sigma_{n}^{2}$, that is, $E\left[n_{k} n_{k-\ell}^{*}\right]=\sigma_{n}^{2} \delta_{k-\ell} \forall k, \ell ;(2)$ the decision delay $\Delta$ is optimized; (3) the SER has been estimated by stopping the simulation after 100 errors occur; (4) each sample at the output of the WL filter is the input of the decision device that performs the symbol-by-symbol ML detection of the transmitted symbol. 
4.1. Fixed Channel. In this section, we compare the performances of the constellation design procedures (26) and (14) in terms of SER. In our simulations, we solve (26) by means of an exhaustive search over $\alpha=n \cdot 0.05$ and $\theta=$ $\pi / 2 \cdot n \cdot 0.05$ : note that in our search we consider $(\alpha, \theta) \in$ $X$, so we consider a finite number of points. On the other hand, we resort to the constrained gradient-based algorithm for solving (14). Since the cost function (13) exhibits local minima, 1000 starting points have been randomly generated according to a uniform distribution. Due to the amount of time required by the computer simulations to determine the solution of (14), we consider, as in [16], the transmission over a two-tap channel $H(z) \triangleq 1+\rho e^{\jmath \phi} z^{-1}$ affected by an additive circularly symmetric white Gaussian noise with variance $\sigma_{n}^{2}$. In our experiments, we have addressed the optimization of the constellation when $K=4$ and $K=8$ for different values of $\rho, \phi$, and $N_{f}$.

Let us first plot in Figure 2 some of the optimum constellations obtained during our simulations when solving the optimization problem (14) over the considered channel model; moreover, we plot the suboptimum constellation utilized to implement our suboptimum strategy and the 8PAM constellation obtained by applying to it the rhombic transformation. As in [2], we have found many local optima, some of them were rotated version of the constellations of Figure 2 while others appeared as their rhombic transformation. For $K=4$ the locally optimum constellation set includes the conventional 4-QAM $(\beta=0)$ and 4$\operatorname{PAM}(\beta=1)$, as well as the 4-QAM subject to a rhombic transformation $(\beta=-0.4+0.3 \mathrm{~J})$; note that such constellations can be obtained by means of a rhombic transformation of the conventional 4-QAM (as also shown in Section 3.2), which has been utilized to implement our suboptimum strategy when $K=4$. For $K=8$, the optimum constellation set includes the noncircular 8-QAM found by Foschini et al. $(\beta=0.12-0.22 \mathrm{~J})$, one of the conventional 8-QAM scheme $(\beta=0)$ called "1-7" 8-QAM [2], the 8-PAM $(\beta=1)$ and the noncircular 8-QAM scheme that we call noncircular 8-QAM. In the following, in order to implement the rhombic-transformation-based constellation-optimization strategy, we resort to the rectangular 8-QAM; we remember that, unlike 4-QAM, such a scheme cannot be transformed into the conventional uniform 8-PAM, but in the nonoptimum nonuniform 8PAM (the optimality of uniform PAM over additive white Gaussian noise has been shown in [23]).

In Figure 3, with reference to the case $K=4$, we have set $\mathrm{SNR} \triangleq 1 / \sigma_{n}^{2}=15 \mathrm{~dB}$ and we have plotted the SERs achieved by both the suboptimum strategy (26) and the optimum strategy (14) versus $\phi$, for $\rho=0.9$ and for different values of $N_{f}\left(N_{f}=4,6\right)$; moreover, for each point of Figure 3 , the constellation typically obtained by the optimum procedure is specified by the letter used to denote it in Figure 2. The results show that the two strategies have the same performance: more specifically, both strategies switch to the 4-PAM when $\phi>\pi / 12$ and outperform the conventional nonadaptive transceiver employing the QPSK modulation jointly with the linear MMSE receiver. Note also that as $\phi \rightarrow$ $\pi / 2$, the chosen value of $N_{f}$ does not affect the performance.

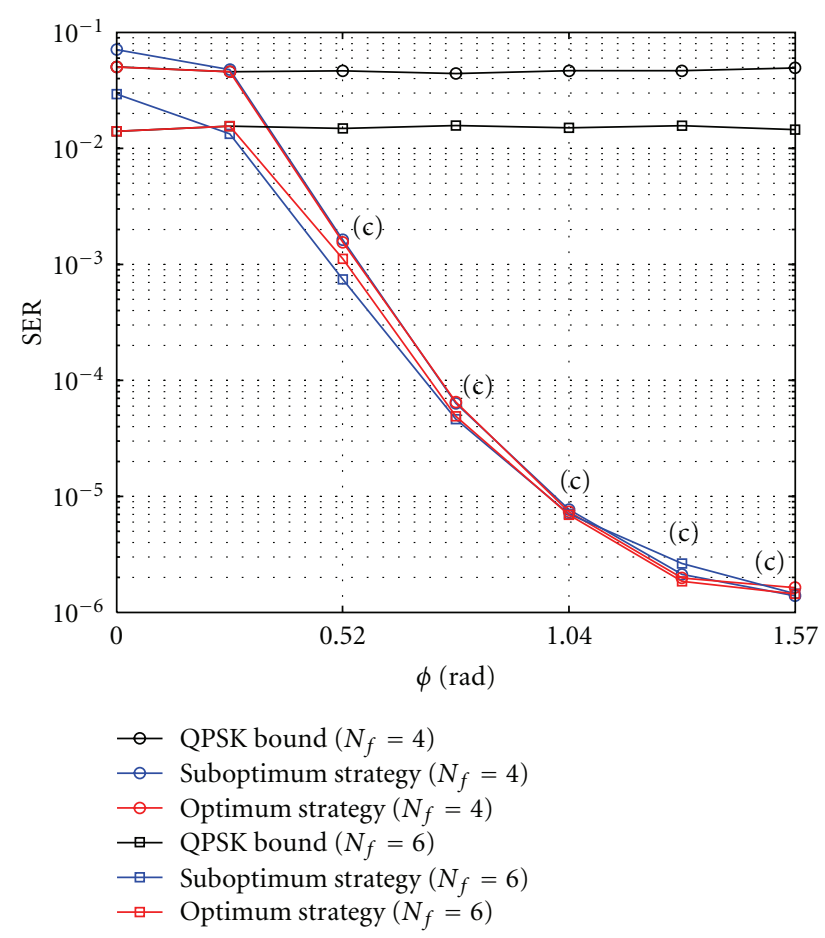

FIgURE 3: Constellation optimization for $K=4$ over fixed channel ( $\rho=0.9$ ); for each point, the letter specifies the constellation (of those in Figure 2) typically obtained.

In the next experiments, we have addressed the constellation optimization when $K=8$; more specifically, in Figures 4 and 5 we have considered the transmission over $H(z)$ when $\rho=0.9$ and $\rho=0.6$, respectively. Figure 4 reports the SER achieved by both the suboptimum strategy and the optimum strategy versus $\phi$ for SNR $=18 \mathrm{~dB}$ and $N_{f}=15$. The optimum strategy provides performance gain over the nonadaptive transceiver employing the conventional rectangular 8-QAM by using the "1-7" 8-QAM and the noncircular 8QAM for smaller values of $\phi$, and, as $\phi>\pi / 6$, by using the 8-PAM. In such a case, the performance difference between the suboptimum strategy and the optimum one is important, especially for large values of $\phi$, since the suboptimum one employs the non-uniform 8-PAM. Such a result was expected since, when $K$ increases, the optimum strategy can exploit a number of degrees of freedom significantly larger than the suboptimum strategy.

Finally, we observe that, when $K=4$, an architecture switching between the 4-QAM and the 4-PAM can provide a good trade-off between performance and complexity. Instead, when $K=8$, the transceiver should switch among the Foschinie All, the noncircular 8-QAM and the 8-PAM.

4.2. Random Channel. In the following simulations, we assume that (i) the channel has memory $v=3$ and its taps $h_{k}$ are randomly generated according to a complex-valued circularly-symmetric zero-mean white Gaussian process with unit variance (i.e., $E\left[\left(\mathfrak{R}\left\{h_{k}\right\}\right)^{2}\right]=E\left[\left(\Im\left\{h_{k}\right\}\right)^{2}\right]=1 / 2$ and $E\left[\mathfrak{R}\left\{h_{k}\right\} \Im\left\{h_{k}\right\}\right]=0$ ); (ii) the WL MMSE equalizer 


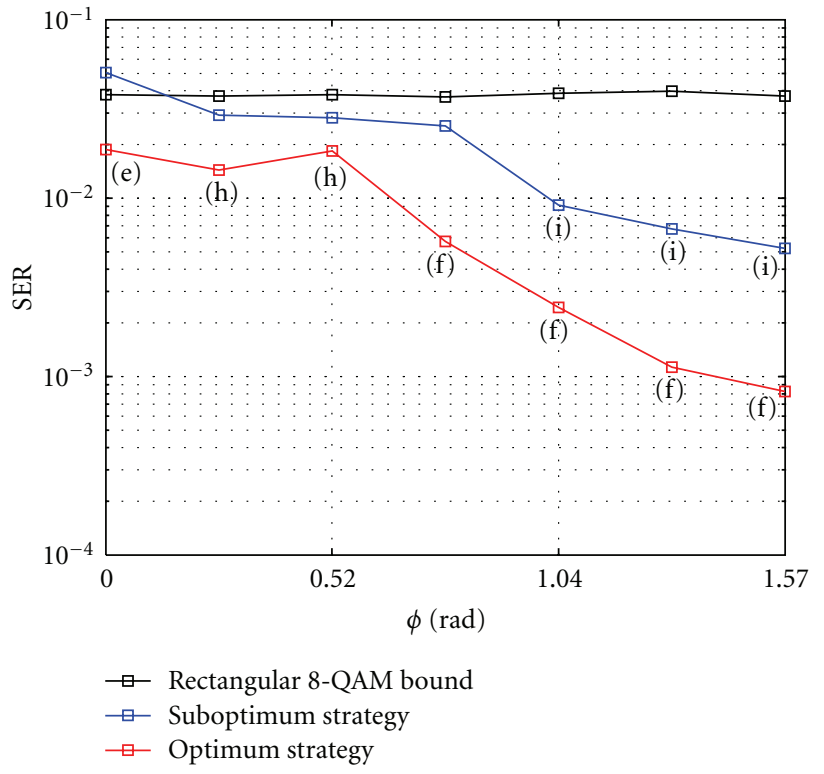

FIgURE 4: Constellation optimization for $K=8$ over fixed channel ( $\rho=0.9$ ); for each point, the letter specifies the constellation (of those in Figure 2) typically obtained.

has $N_{f}=12$ taps; (iii) the results have been averaged over 500 independent channel realizations. We compare the performances achieved by four architectures: (I) the OPTimum-based architecture (OPT-based) that selects $\alpha$ and $\theta$ in order to minimize the symbol error rate (i.e., $P_{e}^{\text {(true) }}(\alpha, \theta)$, instead of $\left.P_{e}^{(\text {low })}(\alpha, \theta)\right)$; (II) the QAM-based architecture adopting the conventional circularly symmetric 4-QAM constellation; (III) the PAM-based architecture utilizing the conventional rotationally variant 4 -PAM $(|\beta|=1$ which corresponds to the maximum WL gain); (IV) the twochoice-based architecture that switches between the 4-QAM and the 4-PAM constellations according to (27). For clarity, we point out that the solution of (26) loses about $0.3 \mathrm{~dB}$ in comparison with the OPT-based one; we consider the OPTbased architecture in order to provide a lower bound to the SER. The OPT-based and the two-choice-based architectures, unlike the QAM-based and the PAM-based ones, require the existence of a feedback channel between the receiver and the transmitter for constellation adaptation; however, the twochoice-based architecture only needs to transmit a binary information on such feedback channel.

In Figure 6, the SERs of the considered architectures are plotted versus the SNR (in $\mathrm{dB}$ ). The OPT-based architecture outperforms all the others and provides an SNR-gain over the nonoptimized architectures of almost $3 \mathrm{~dB}$ for a SER $=10^{-3}$. Interestingly, the two-choice-based architecture performs well loosing only $0.8 \mathrm{~dB}$ in comparison with the OPT-based one. Let us also note that the PAM-based architecture performs poorly for low SNR, but, as the SNR increases, it outperforms the QAM-based one.

In the next experiment, we compare the considered architectures by evaluating their capability to guarantee the required quality of service (QoS). More specifically, in

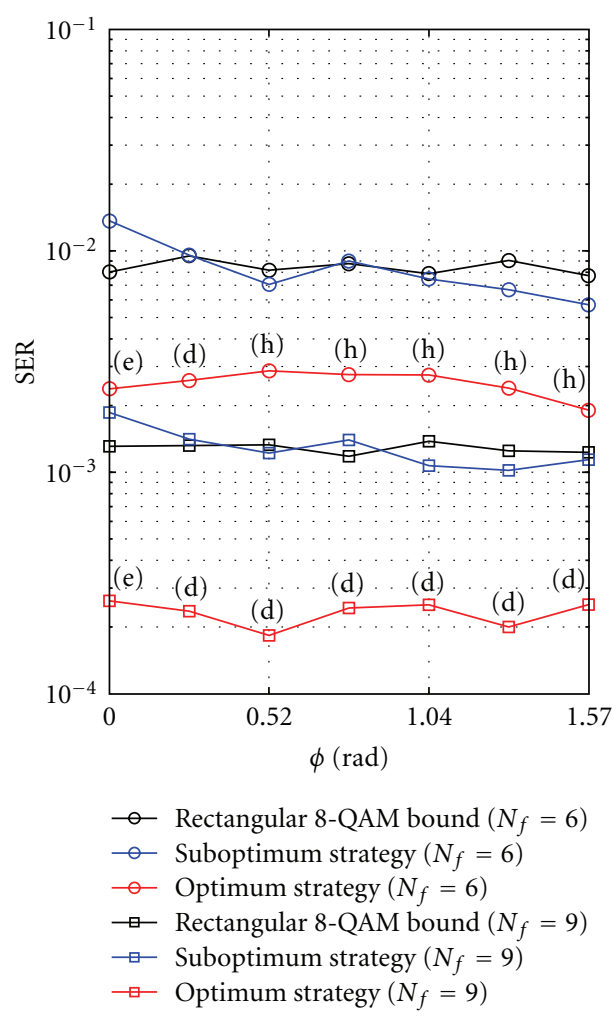

Figure 5: Constellation optimization for $K=8$ over fixed channel ( $\rho=0.6$ ); for each point, the letter specifies the constellation (of those in Figure 2) typically obtained.

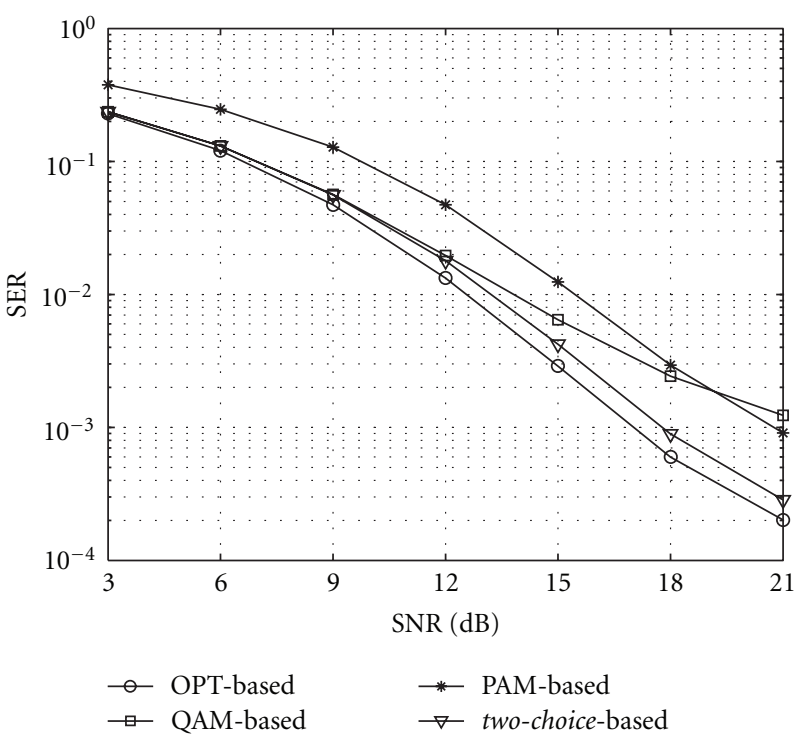

FIGURE 6: SER of the considered architectures versus SNR.

Table 1, we report the percentages of the channels over which the SNR required to achieve the target SER (assumed to be $10^{-2}, 10^{-3}, 10^{-4}$ ) is not larger than $21 \mathrm{~dB}$. Moreover, Figure 7 reports the probability, say $P_{\mathcal{E}}$, that each architecture loses $\varepsilon \mathrm{dB}$ in comparison with the OPT-based one for a given 


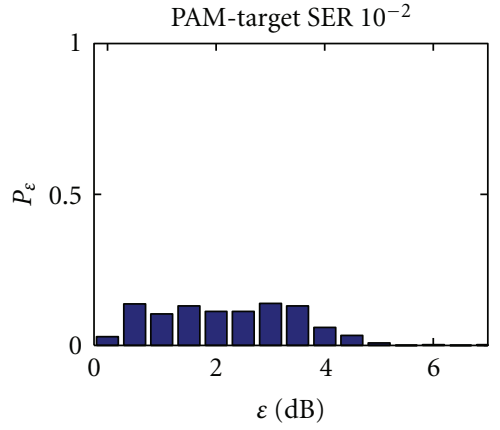

(a)

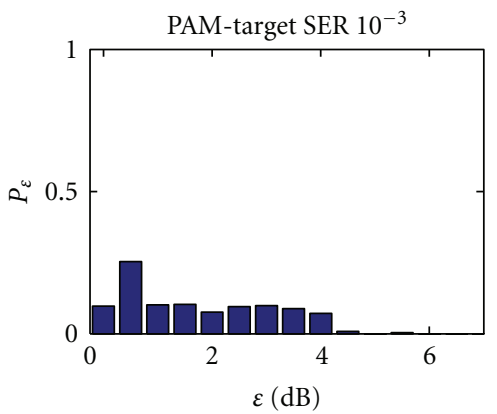

(d)

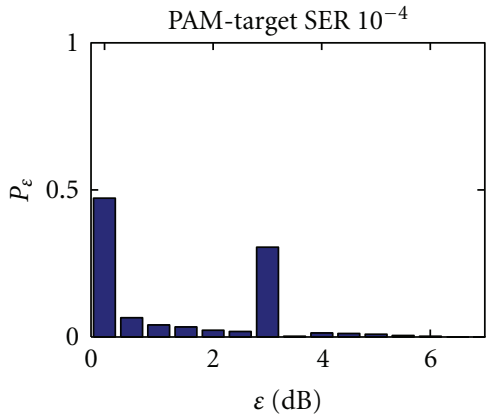

(g)

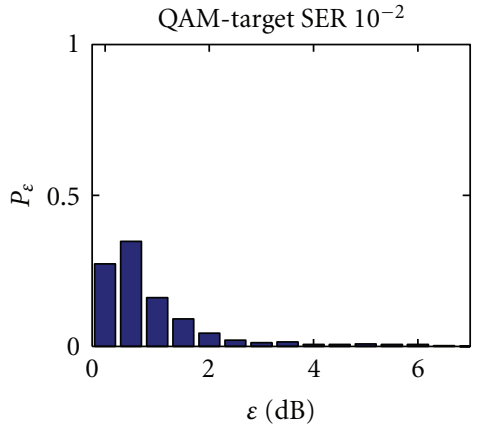

(b)

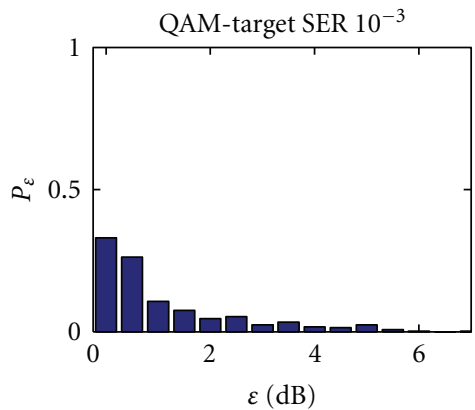

(e)

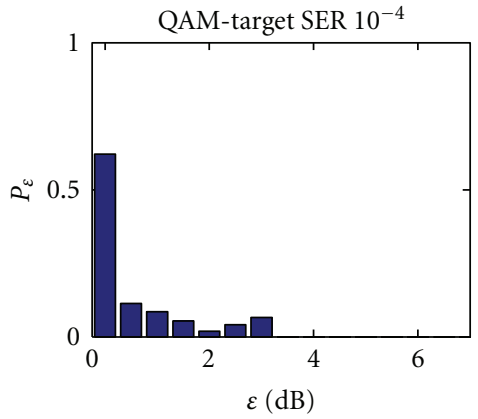

(h)

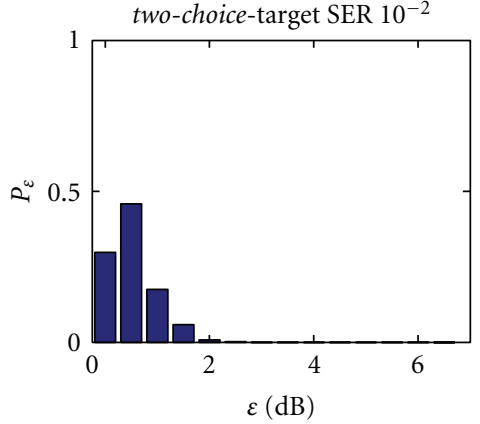

(c)

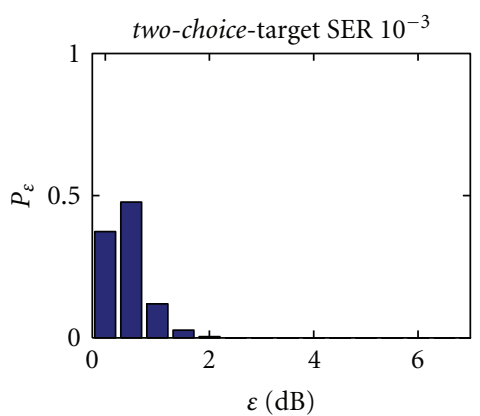

(f)

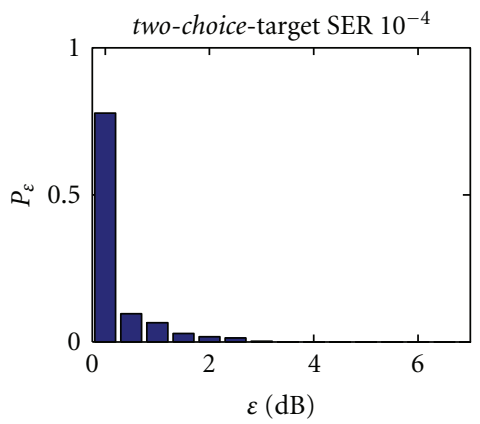

(i)

FIGURE 7: Loss in $\mathrm{dB}$ of the PAM-based, QAM-based, and two-choice-based architectures with respect to the OPT-based one for several target SER.

target SER under the condition that the SNR is not larger than $21 \mathrm{~dB}$.

The results show the following:

(i) The PAM-based architecture is robust with respect to the communication environment since it often achieves the target SER. This is mainly due to the improved capabilities of the WL equalizer when the transmitted and the received signals are rotationally variant. However, it requires a larger SNR in comparison with the OPT-based architecture to compensate for the reduction of the dimension of the signal space. Note also that such an SNR loss, which is uniformly distributed between $0 \mathrm{~dB}$ and $4 \mathrm{~dB}$ when the target SER is $10^{-2}$ and $10^{-3}$, assumes often two specific values $(0 \mathrm{~dB}$ and $3 \mathrm{~dB})$ for a target SER equal to $10^{-4}$. In practice, the PAM architecture achieves optimum performance on $50 \%$ of the channels where the linear equalizer performs unsatisfactorily and the WL processing gain, specific to rotationally variant constellations, compensates for the smaller minimum-distance of the PAM constellation.

(ii) The QAM-based architecture is not robust with respect to the communication environment. When it is able to achieve the target SER, it requires a limited amount of excess SNR over the OPT-based architecture; nevertheless, it is unable to achieve the target SER of $10^{-4}$ on $37 \%$ of the channels. This is due to the circular symmetry of the constellation that does not allow one to improve by means of the WL processing the unsatisfactory performance of the linear equalizer.

(iii) The two-choice-based architecture is particularly simple and robust since it combines the advantages of both PAM and QAM constellations. 
TABle 1: Percentage of channels over which the target SER is achieved.

\begin{tabular}{lcccc}
\hline Target SER & OPT-based & QAM-based & PAM-based & $\begin{array}{c}\text { Two-choice- } \\
\text { based }\end{array}$ \\
\hline $10^{-2}$ & $100 \%$ & $97 \%$ & $98 \%$ & $99 \%$ \\
$10^{-3}$ & $97 \%$ & $83 \%$ & $95 \%$ & $96 \%$ \\
$10^{-4}$ & $94 \%$ & $63 \%$ & $89 \%$ & $92 \%$ \\
\hline
\end{tabular}

\section{Conclusions}

We have addressed the problem of constellation optimization for the WL MMSE equalizer. By modeling the residual disturbance at the output of the WL equalizer as a white Gaussian (possibly rotationally variant) process, we have singled out constellation-design methods which minimize an upper bound of the symbol error rate. The first method exploits all the degrees of freedom $(2 K)$ associated to the $K$-order constellation exhibiting, therefore, an unaffordable computational complexity for high-order constellations. To overcome such a problem, a second design method based on a rhombic transformation of a fixed alphabet of order $K$ is proposed. It performs the optimization of only two parameters (instead of $2 K$ ) leading to a huge reduction of the computational complexity for large $K$. For low-order constellations, the simulation results show that the two techniques are practically equivalent in terms of symbol error rate; moreover, they also show that a WL MMSE transceiver with constellation adaptation is clearly superior to the same equalizer with fixed constellation. Finally, for $K=4$, it has been shown that the method that switches between a real-valued and a complex-valued constellation exhibits a limited performance loss versus the optimum adaptation scheme, while it achieves a strong reduction of the computational complexity and it requires to feed back to the transmitter only a binary information.

\section{References}

[1] R. Lucky and J. Hancock, "On the optimum performance of $\mathrm{N}$-ary systems having two degrees of freedom," IRE Transactions on Communications Systems, vol. 10, pp. 185-192, 1962.

[2] G. J. Foschini, R. D. Gitlin, and S. B. Weinstein, "Optimization of two-dimensional signal constellations in the presence of Gaussian noise," IEEE Transactions on Communications, vol. 22, no. 1, pp. 28-38, 1974.

[3] P. Chevalier and F. Pipon, "New insights into optimal widely linear array receivers for the demodulation of BPSK, MSK, and GMSK signals corrupted by noncircular interferencesapplication to SAIC," IEEE Transactions on Signal Processing, vol. 54, no. 3, pp. 870-883, 2006.

[4] D. P. Palomar, J. M. Cioff, and M. A. Lagunas, "Joint TxRx beamforming design for multicarrier MIMO channels: a unified framework for convex optimization," IEEE Transactions on Signal Processing, vol. 51, no. 9, pp. 2381-2401, 2003.
[5] F. Xu, T. N. Davidson, J.-K. Zhang, and K. M. Wong, "Design of block transceivers with decision feedback detection," IEEE Transactions on Signal Processing, vol. 54, no. 3, pp. 965-978, 2006.

[6] Y. Jiang, J. Li, and W. W. Hager, "Uniform channel decomposition for MIMO communications," IEEE Transactions on Signal Processing, vol. 53, no. 11, pp. 4283-4294, 2005.

[7] A. Scaglione, P. Stoica, S. Barbarossa, G. B. Giannakis, and H. Sampath, "Optimal designs for space-time linear precoders and decoders," IEEE Transactions on Signal Processing, vol. 50, no. 5, pp. 1051-1064, 2002.

[8] B. Picinbono, "On circularity," IEEE Transactions on Signal Processing, vol. 42, no. 12, pp. 3473-3482, 1994.

[9] K. Kuchi and V. K. Prabhu, "Interference cancellation enhancement through generalized widely linear equalization in QAM systems," IEEE Transactions on Wireless Communications, vol. 8, no. 4, pp. 1585-1590, 2009.

[10] B. Picinbono and P. Chevalier, "Widely linear estimation with complex data," IEEE Transactions on Signal Processing, vol. 43, no. 8, pp. 2030-2033, 1995.

[11] K. Kuchi and V. K. Prabhu, "Performance evaluation for widely linear demodulation of PAM/QAM signals in the presence of Rayleigh fading and co-channel interference," IEEE Transactions on Communications, vol. 57, no. 1, pp. 183193, 2009.

[12] W. H. Gerstacker, R. Schober, and A. Lampe, "Equalization with widely linear filtering," in Proceedings of the IEEE International Symposium on Information Theory (ISIT '01), $\mathrm{p}$. 265, June 2001.

[13] A. Mirbagheri, K. N. Plataniotis, and S. Pasupathy, "An enhanced widely linear CDMA receiver with OQPSK modulation," IEEE Transactions on Communications, vol. 54, no. 2, pp. 261-272, 2006.

[14] A. Parihar, L. Lampe, R. Schober, and C. Leung, "Equalization for DS-UWB systems-part II: 4BOK modulation," IEEE Transactions on Communications, vol. 55, no. 8, pp. 15251535, 2007.

[15] G. Gelli, L. Paura, and A. R. P. Ragozini, "Blind widely linear multiuser detection," IEEE Communications Letters, vol. 4, no. 6, pp. 187-189, 2000.

[16] W. H. Gerstacker, R. Schober, and A. Lampe, "Receivers with widely linear processing for frequency-selective channels," IEEE Transactions on Communications, vol. 51, no. 9, pp. 15121523, 2003.

[17] A. Parihar, L. Lampe, R. Schober, and C. Leung, "Equalization for DS-UWB systems-part I: BPSK modulation," IEEE Transactions on Communications, vol. 55, no. 6, pp. 11641173, 2007.

[18] D. Mattera, L. Paura, and F. Sterle, "Widely linear MMSE equaliser for MIMO linear time-dispersive channel," Electronics Letters, vol. 39, no. 20, pp. 1481-1482, 2003.

[19] A. Lampe and M. Breiling, "Asymptotic analysis of widely linear MMSE multiuser detection-complex vs real modulation," in Proceedings of the IEEE Information Theory Workshop, pp. 55-57, September 2001.

[20] J. B. Anderson, Digital Transmission Engineering, IEEE Press, New York, NY, USA, 2nd edition, 2005.

[21] Y.-P. Lin and S.-M. Phoong, "Optimal ISI-free DMT transceivers for distorted channels with colored noise," IEEE Transactions on Signal Processing, vol. 49, no. 11, pp. 27022712, 2001. 
[22] D. P. Palomar and S. Barbarossa, "Designing MIMO communication systems: constellation choice and linear transceiver design," IEEE Transactions on Signal Processing, vol. 53, no. 10, pp. 3804-3818, 2005.

[23] A. M. Makowski, "On the optimality of uniform pulse amplitude modulation," IEEE Transactions on Information Theory, vol. 52, no. 12, pp. 5546-5549, 2006. 